\title{
Expert panel consensus recommendations for home blood pressure monitoring in Asia: the Hope Asia Network
}

\author{
Sungha Park ${ }^{1}$ Peera Buranakitjaroen ${ }^{2}$ Chen-Huan Chen ${ }^{3} \cdot$ Yook-Chin Chia ${ }^{4,5} \cdot$ Romeo Divinagracia $^{6}$. \\ Satoshi Hoshide ${ }^{7} \cdot$ Jinho Shin ${ }^{8}$. Saulat Siddique ${ }^{9} \cdot J^{\prime}$ orge Sison ${ }^{10}$ - Arieska Ann Soenarta ${ }^{11}$. \\ Guru Prasad Sogunuru ${ }^{12,13} \cdot$ Jam Chin Tay ${ }^{14} \cdot$ Yuda Turana ${ }^{15} \cdot$ Ji-Guang Wang ${ }^{16} \cdot$ Yuqing Zhang ${ }^{17}$. \\ Kazuomi Kario $\mathbb{1}^{7}$ on behalf of the HOPE Asia Network
}

Received: 29 June 2017 / Revised: 12 September 2017 / Accepted: 4 October 2017 / Published online: 31 January 2018

(c) The Author(s) 2018. This article is published with open access

\begin{abstract}
Hypertension is the leading cause of mortality throughout Asia. Home blood pressure monitoring has the potential to improve hypertension control and is a useful adjunct to conventional office blood pressure measurements due to its diagnostic accuracy and prognostic value in predicting cardiovascular outcomes. At present, there are no region-specific guidelines addressing the use of home blood pressure monitoring in Asia. Therefore, an expert panel was convened to address the use of home blood pressure monitoring and develop key recommendations to help guide clinical practice throughout the Asia region. The resulting recommendations support the use of home blood pressure monitoring with a validated device as an accurate adjunct for diagnosing hypertension and predicting cardiovascular outcome. Diagnosis and treatment of hypertension should still be guided by conventional office/clinic blood pressure measurements. The expert panel encourages the incorporation of home blood pressure monitoring into local clinical guidelines and offers practical recommendations to ensure continuity of care where a validated home blood pressure device is not available.
\end{abstract}

HOPE Asia Network: (Hypertension Cardiovascular Outcome Prevention and Evidence in Asia)

Kazuomi Kario

kkario@jichi.ac.jp

1 Division of Cardiology, Cardiovascular Hospital, Yonsei Health System, Seoul, Korea

2 Department of Medicine, Faculty of Medicine Siriraj Hospital, Mahidol University, Bangkok 10700, Thailand

3 Department of Medicine, Faculty of Medicine, National YangMing University, Taipei, Taiwan

4 Department of Primary Care Medicine, Faculty of Medicine, University of Malaya, Kuala Lumpur, Malaysia

5 Sunway lnstitute for Healthcare Development, Sunway University, Selangor Darul Ehsan, Malaysia

6 University of the East Ramon Magsaysay Memorial Medical Center Inc, Quezon City, Philippines

7 Division of Cardiovascular Medicine, Department of Medicine, Jichi Medical University School of Medicine, Tochigi, Japan

8 Faculty of Cardiology Service, Hanyang University Medical Center, Seongdong, Seoul, Korea

9 Punjab Medical Center, Jail Road, Lahore, Pakistan
10 Section of Cardiology, Department of Medicine, Medical Center Manila, Manila, Philippines

11 Department of Cardiology and Vascular Medicine, University of Indonesia-National Cardiovascular Center, Harapan Kita, Jakarta, Indonesia

12 Apollo Hospitals, Chennai, India

13 College of Medical Sciences, Kathmandu University, Bharatpur, Nepal

14 Department of General Medicine, Tan Tock Seng Hospital, Singapore, Singapore

15 Department of Neurology, Faculty of Medicine, Atma Jaya Catholic University of Indonesia, Jakarta, Indonesia

16 Department of Hypertension, Centre for Epidemiological Studies and Clinical Trials, The Shanghai Institute of Hypertension, Shanghai Key Laboratory of Hypertension, Ruijin Hospital, Shanghai Jiaotong University School of Medicine, Shanghai, China

17 Divisions of Hypertension and Heart Failure, Fu Wai Hospital, Chinese Academy of Medical Sciences and Peking Union Medical College, Beijing, China 


\section{Introduction}

Hypertension is the leading preventable cause of premature mortality worldwide [1,2]. Consistent with the global trend, hypertension is also the single most attributable cause for mortality in Asia where some countries continue to experience increasing rates of cardiovascular disease (CVD) mortality along with low disease control and awareness rates [3, 4]. Several factors contribute to the high burden of hypertension in Asia. These include an increasing influence of Western lifestyle, an ageing population, high salt intake, an obesity epidemic, and-compared with Western countries-a higher morbidity and mortality from cerebrovascular disease [5, 6]. Effective prevention and control of hypertension remains a challenge in the region, with barriers including cultural practices that promote unhealthy behaviours and misconceptions about hypertension, high smoking rates, high out-of-pocket treatment costs, and poor treatment adherence [3].

Dramatic reductions in CVD mortality are possible, as shown by the $37 \%$ reduction achieved in Korea between 2000 and 2012 with an accompanying mean reduction in systolic blood pressure (SBP) among adults of around $10 \mathrm{~mm} \mathrm{Hg}$ [4]. This level of blood pressure (BP) reduction was attributed to improved hypertension awareness, treatment, and control rates. However, effective screening and diagnosis are also critical components of hypertension management and CVD prevention, and there is growing recognition for the role of out-of-office BP monitoring [7]. Home blood pressure monitoring (HBPM) is a convenient and standardised tool recommended by several national and international guidelines as an adjunct to conventional office BP measurements due to its diagnostic accuracy and prognostic value in predicting cardiovascular outcomes [8-10]. However, many patients in Asia are yet to receive this level of intervention [11].

Herein, we summarise the evidence-based consensus recommendations of an expert panel on HBPM convened for Asia. It is anticipated that these recommendations will help guide clinical practice in the region, with the aim of optimising BP monitoring and therefore improving the management of hypertension.

\section{Materials and methods}

The panel's consensus recommendations were the output of a meeting convened in Seoul, Korea, on 24 September 2016, and based on a review of evidence from meta-analyses, randomised controlled trials (RCTs), and other relevant data on HBPM. In order to ensure applicability of any findings to diverse Asian populations, emphasis was given to data sourced from the region. However, where evidence was based on studies conducted outside Asia, the expert panel used their clinical expertise and judgement to analyse the findings in the context of hypertension management in the region.

\section{Results}

The expert panel identified key items relating to HBPM for discussion and consensus statements and their scientific evidence base are discussed herein. Specific recommendations for each item are summarised in Table 1.

\section{Accuracy of home BP for diagnosis of hypertension}

Initial diagnosis of hypertension conventionally involves clinic- or office-based BP measurements. Discrepancies are often noted, however, between BP measurements taken in and out of the office. Office-based BP measurements are usually higher compared with ambulatory blood pressure monitoring (ABPM). The magnitude of this discrepancy was assessed in a sample of almost 13,000 treated patients with hypertension [12]. Whereas $23.6 \%$ of patients were considered to have controlled hypertension based on office BP measurements, $51.6 \%$ were controlled according to daytime ABPM. Among patients with an average daytime ambulatory $\mathrm{BP}$ of $<135 / 85 \mathrm{~mm} \mathrm{Hg}, 33.4 \%$ were considered to be resistant based on an office BP measurement of $\geq 140$ / $90 \mathrm{~mm} \mathrm{Hg}$. Conversely, among patients with uncontrolled hypertension based on an average daytime ambulatory BP measurement of $\geq 135 / 85 \mathrm{~mm} \mathrm{Hg}, 5.4 \%$ were considered to be controlled based on an office BP measurement of $<140 /$ $90 \mathrm{~mm} \mathrm{Hg}$.

Similar to ABPM, HBPM provides multiple BP readings over several days. Its use continues to gain acceptance as data accumulate and accurate equipment becomes more widely available. The relative effectiveness of clinic BP measurements and HBPM was compared with ABPM for the diagnosis of hypertension in a systematic review [13]. Compared with ambulatory monitoring thresholds of 135/ $85 \mathrm{~mm} \mathrm{Hg}$, clinic measurements over 140/90 mm Hg had a mean sensitivity and specificity of 74.6 and $74.6 \%$, respectively. In comparison, home measurements over a threshold of $135 / 85 \mathrm{~mm} \mathrm{Hg}$ had a mean sensitivity and specificity of 85.7 and $62.4 \%$, respectively. Based on ABPM as the reference standard, the investigators concluded that neither clinic nor home measurement on their own had sufficient sensitivity or specificity to be recommended as a single diagnostic test, with both resulting in overdiagnosis. However, they also acknowledged the improved sensitivity of HBPM compared with office measurements and the suitability of HBPM for excluding hypertension given its greater availability compared with ambulatory monitoring. 
Table 1 Summary of recommendations for home blood pressure monitoring in Asia

Recommendation

1. HBPM is an accurate adjunct for diagnosing hypertension when a validated device is used and the measurement is performed correctly.

2. Method of measuring home BP:

Class of

recommendation

I

Level of

evidence

- Sitting BP after 2 min of rest

- Wearing light clothes while taking the reading is allowed

- At least 2 readings, with a 1-min interval, twice daily, for at least 3 days, but preferably 7 days

- Morning: within $1 \mathrm{~h}$ after waking, after urination, before breakfast, and before drug intake

- Evening: before going to bed

- Elevated BP is shown by a mean reading of $\geq 135 / 85 \mathrm{~mm} \mathrm{Hg}$

3a. HBPM is a better predictor of cardiovascular outcome than office BP. When there is a discrepancy of diagnosis between office and home BP, a home BP-based diagnosis should have priority and, when possible, be confirmed by ABPM.

3b. Morning hypertension is a better predictor of prognosis than clinic BP.

4. Antihypertensive treatment strategies should target a home BP level of $<135 / 85 \mathrm{~mm} \mathrm{Hg}$.

Strict antihypertensive treatment targeting a home systolic BP level of $<125 \mathrm{~mm} \mathrm{Hg}$ may have benefit in high-risk Asian hypertensive patients, especially those with diabetes or chronic kidney disease, and/or cardiovascular disease.

5a. The diagnosis and treatment of hypertension should still be guided by office BP readings where HBPM is not readily available. However, HBPM can improve compliance when combined with active intervention, and thus improve BP control compared with current standard care alone.

5b. Self-monitoring and self-titration may be feasible if carefully monitored by healthcare professionals and help to improve blood pressure control. However, local policies must be adhered to as self-titration is not recommended in certain countries.

6. Titration should be based on targeting a mean home BP of $<135 / 85 \mathrm{~mm} \mathrm{Hg}$. However, in cases of high morning BP and normal evening BP, uptitration of drug treatment should be considered even if mean home BP is $<135 / 85 \mathrm{~mm} \mathrm{Hg}$. HBPM may aid chronotherapy of hypertension by helping identify those patients who experience isolated morning hypertension.

7. HBPM may be incorporated into local clinical hypertension guidelines.

8. Information and communication technology (ICT)-based HBPM may be beneficial, especially for patients who live in remote Asian geographical locations. The expert panel agreed that that telemonitoring of home BP, which requires active participation by patients, may have an important role in clinical practice in the near future in Asia.

9. A validated brachial BP measuring oscillometric device should be used for measuring home BP.

Where this is not feasible, the brachial BP measuring oscillometric device of choice should be calibrated every 6-12 months.

Classes of recommendations:

Class I: evidence and/or general agreement that a given treatment or procedure is beneficial, useful, effective (is recommended/is indicated);

Class IIa: weight of evidence/opinion is in favour of usefulness/efficacy (should be considered);

Class IIb: usefulness/efficacy is less well established by evidence/opinion (may be considered);

Class III: evidence or general agreement that the given treatment or procedure is not useful/effective, and in some cases may be harmful (is not recommended).

Levels of evidence:

A: data derived from multiple randomised clinical trials or meta-analyses;

B: data derived from a single randomised clinical trial or large nonrandomised studies;

C: consensus of opinion of the experts and/or small studies, retrospective studies, registries

$B P$ blood pressure, $H B P M$ home blood pressure monitoring 


\section{Recommendation 1}

HBPM is an accurate adjunct for diagnosing hypertension when a validated device is used and the measurement is performed correctly, and leads to more appropriate targeting of treatment rather than relying on clinic measurements alone (Class I, Level of Evidence B).

\section{Method of measuring home BP}

Numerous guidelines and recommendations for home BP measurements have been published globally, including the European Society of Hypertension (ESH) practice guidelines for HBPM [14], the Japanese Society of Hypertension (JSH) guidelines [9], the China consensus document on HBPM [15], and the Korean Society of Hypertension guidelines [10]. All these guidelines have subtle differences, perhaps reflecting differences in local clinical practice, practical implications of BP measurement, and cultural considerations. For example, these guidelines typically recommend taking a sitting BP measurement after 5 min of rest, but the JSH guidelines recommend a rest period of 1-2 min. In the Japanese and Korean guidelines, an HBPM observation period as long as possible is recommended, while the European and Chinese guidelines specify an observation period of at least 3 days and 5 days, respectively.

The Finn-Home study evaluated home BP measurements in an unselected Finnish population of more than 2000 subjects aged 45 to 74 years to determine an optimal schedule [16]. Prognostic data from this study showed that home BP measurements taken twice in the morning and again in the evening, preferably for a period of 7 days and for a minimum of 3 days, provided the best estimation of a patient's BP level. The 10-year follow-up results from the Ohasama study of almost 1500 Japanese people found that the predictive value of HBPM increased progressively with the number of days of measurements [17]. When multiple days of measurements were taken, HBPM more reliably predicted the risk of stroke per $10 \mathrm{~mm} \mathrm{Hg}$ increase in SBP; with a mean of 25 days of measurements, HBPM predicted a $35 \%$ increased risk of stroke per $10 \mathrm{~mm} \mathrm{Hg}$ increase compared with a predicted risk of $20 \%$ for a 2-day HBPM period.

\section{Recommendation 2}

The expert panel recommends conditions of monitoring that include taking the seated BP after 2 min of rest preferably without smoking, alcohol consumption, or exercise for the previous $30 \mathrm{~min}$, and permitting the placement of the arm cuff over light clothing. At initiation of HBPM, before follow-up appointments and after adjustments to treatment (e.g., dose titration), at least two readings-with a 1-min interval-per occasion should be taken twice daily for a minimum of 3 days and preferably 7 days. For long-term HBPM under normal circumstances, one reading per occasion may be more convenient and sustainable for patients. Morning BP measurements should be taken within $1 \mathrm{~h}$ of waking, after urination, and before breakfast and drug intake. The evening BP measurements should be taken before going to bed. An elevated BP is considered to be a mean home BP of $\geq 135 / 85 \mathrm{~mm} \mathrm{Hg}$. Morning BP is a better measure than evening BP to exclude the effects of different evening activities in Asia, and to more accurately determine the pathophysiology of BP variability (Class I, Level of Evidence B).

\section{Predicting cardiovascular outcome: office BP or HBPM?}

The two major categories of discordance between home and office/clinic BP measurements are white-coat hypertension involving elevated office/clinic BP with normal ambulatory or home BP measurements, and masked hypertension involving elevated ambulatory or home BP measurements with normal office/clinic BP [18]. HBPM is not influenced by observer bias and the white-coat effect, and delivers greater reproducibility than casual BP screening. The prognostic benefits of HBPM have also been well established and there is relevant regional evidence supporting the use of HBPM to predict cardiovascular outcome. In the Ohasama study of more than 1700 subjects aged $\geq 40$ years who were followed for a mean of 6.6 years, home BP measurement had a stronger predictive power for mortality than office-based screening BP measurement [19]. Another Japanese study used data from the HONEST study to investigate the relationship between on-treatment morning home BP and incidence of cardiovascular events [20]. The HONEST study was a prospective, observational study of more than 21,000 patients with essential hypertension who received antihypertensive medication over a mean followup of 2 years. The risk of a major cardiovascular event was significantly higher in patients with on-treatment morning systolic home BP $\geq 145$ to $<155 \mathrm{~mm} \mathrm{Hg}$ (hazard ratio (HR), 1.83 ; $95 \%$ confidence interval (CI), 1.12 to 2.99 ) and $\geq 155$ $\mathrm{mm} \mathrm{Hg}$ (HR, 5.03; 95\% CI, 3.05 to 8.31) compared with $<125 \mathrm{~mm} \mathrm{Hg}$. Morning systolic home BP associated with minimum risk was $124 \mathrm{~mm} \mathrm{Hg}$. Additionally, a recent analysis from the HONEST study demonstrated that morning systolic home BP $\geq 155 \mathrm{~mm} \mathrm{Hg}$ was associated with an HR of 6.24 (95\% CI, 2.82 to 13.84) for predicting future coronary artery disease compared with an HR of 3.51 (95\% CI: $1.71-7.20$ ) for clinic SBP of $\geq 160 \mathrm{~mm} \mathrm{Hg}$ [21].

The expert panel also considered other studies that evaluated the prognostic role of HBPM. Among these, the 
Japan Morning Surge-Home Blood Pressure study aimed to determine the optimal time schedule for HBPM to best predict stroke and coronary artery disease in over 4000 Japanese people with a history of, or risk factors for, CVD [22]. During a mean follow-up of 4 years, morning SBP was shown to improve the discrimination of incident stroke beyond traditional risk factors including office SBP; as such, the authors concluded that morning home SBP itself should be evaluated to ensure best stroke prediction in clinical practice. The Finn-Home study found that in more than 2000 randomly selected subjects followed for a mean of 6.8 years, home BP measurements was a stronger predictor of cardiovascular risk than office BP measurements [23]. When both home and office BP measurements were included in predictive models, only home BP predicted cardiovascular events with an $\mathrm{HR}$ per $10 / 5 \mathrm{~mm} \mathrm{Hg}$ increase of $1.22 / 1.15$ (95\% CI, 1.09 to $1.37 / 1.05$ to 1.26 ). SBP was the sole predictor of total mortality (HR, 1.11; $95 \%$ CI, 1.01 to 1.23$)$.

\section{Recommendation 3a}

The expert panel confirmed its view that HBPM is a better predictor of cardiovascular outcome than office BP. When there is a discrepancy of diagnosis between office and home $\mathrm{BP}$, a home BP-based diagnosis should have priority and, when possible, be confirmed by ABPM (Class I, Level of Evidence B).

\section{Recommendation $3 \mathrm{~b}$}

Morning hypertension, assessed by either HBPM or ABPM, is a better predictor of prognosis than clinic BP (Class I, Level of Evidence B).

\section{Recommendation 4}

For antihypertensive treatment strategies based on HBPM, the target home BP level should be $<135 / 85 \mathrm{~mm} \mathrm{Hg}$ (Class I, Level of Evidence B).

Strict antihypertensive treatment targeting a home systolic BP level of $<125 \mathrm{~mm} \mathrm{Hg}$ may have benefit in high-risk Asian hypertensive patients, especially those with diabetes or chronic kidney disease, and/or CVD (Class IIa, Level of Evidence B).

\section{Improved BP control and prognosis: office BP or HBPM?}

Despite the positioning of HBPM in international guidelines, 2013 guidelines of the ESH and the European Society of Cardiology (ESC) recommend that the diagnosis and treatment of hypertension should still be based on office BP measurements [24]. The same guidelines recommend that ABPM and HBPM should be utilised to assess treatment, white-coat hypertension, masked hypertension, and hypertension in pregnancy. This view was endorsed by the findings of a trial conducted in Belgium and Ireland involving 400 patients with an untreated diastolic BP of $\geq 95 \mathrm{~mm} \mathrm{Hg}$ who were randomised to home- or clinic BPbased treatment and followed for 1 year [25]. In this study, adjustment of antihypertensive treatment based on home BP measurements led to less intensive drug treatment and lower costs than office BP measurements, but also resulted in less BP control with no differences in general well-being or left ventricular mass. Home BP measurement was effective at identifying patients with white-coat hypertension. More patients in the home BP group stopped treatment compared with the office BP group (25.6 versus $11.3 \%$, respectively; $P<0.001$ ), with no difference in progression to sustained multiple drug treatment (38.7 versus $45.1 \% ; P=0.14$ ). Overall, the findings supported an approach in which HBPM is used as a complement to conventional office BP measurements for the adjustment of antihypertensive treatment.

The findings of the Staessen study reinforce the importance of treatment adherence. The relationship between HBPM and medication compliance in patients with hypertension was evaluated in a 6-month prospective study in Spain involving 250 patients with newly diagnosed or uncontrolled hypertension [26]. Following randomisation to HBPM or standard care, medication compliance defined as drug consumption of $80-110 \%$ was 92 and $74 \%$, respectively $(P=0.0001)$, suggesting that HBPM is effective at improving compliance. The number needed to treat to avoid one case of noncompliance was 5.6 patients. Moreover, a systematic review and meta-analysis that included 9446 participants randomised to home or clinic $\mathrm{BP}$ monitoring concluded that the former is associated with less therapeutic inertia [27]. The investigators additionally reported that compared with clinic BP measurement, both systolic and diastolic BP improved with home-based BP monitoring. HBPM was associated with more frequent medication reductions (relative risk (RR), 2.02; 95\% CI, 1.32 to 3.11 ) and the RR of therapeutic inertia was reduced by $18 \%$ (RR, $0.82 ; 95 \%$ CI, 0.68 to 0.99 ).

A recent RCT evaluating the effect of HBPM with selftitration of antihypertensive medication compared with usual care warrants consideration. This primary care study involved 552 patients aged $\geq 35$ years with a history of stroke, coronary heart disease, diabetes, or chronic kidney disease with a baseline BP of $\geq 130 / 80 \mathrm{~mm} \mathrm{Hg}$ [28]. The target office BP was $130 / 80 \mathrm{~mm} \mathrm{Hg}$ compared with a target home BP of $120 / 75 \mathrm{~mm} \mathrm{Hg}$. After 12 months, mean BP decreased to $128.2 / 73.8 \mathrm{~mm} \mathrm{Hg}$ in the home BP group and to $137.8 / 76.3 \mathrm{~mm} \mathrm{Hg}$ in the control group, a baseline- 
corrected treatment difference of 9.2/3.4 mm $++\mathrm{Hg}(95 \%$ CI, 5.7 to 12.7 systolic; $95 \%$ CI, 1.8 to 5.0 diastolic). These results suggest that self-monitoring with self-titration may be feasible if patients are carefully monitored by healthcare professionals.

\section{Recommendation $5 \mathrm{a}$}

The expert panel recommends that the diagnosis and treatment of hypertension should still be guided by office BP readings in areas where HBPM is not readily available in Asia. However, the panel also acknowledge that HBPM can improve adherence when combined with active intervention, and thus improve BP control compared with current standard care alone (Class I, Level of Evidence B).

\section{Recommendation $5 \mathrm{~b}$}

Self-monitoring and self-titration may be feasible if carefully monitored by healthcare professionals and help improve BP control. However, local policies must be adhered to as self-titration is not recommended in certain countries (Class IIb, Level of Evidence B).

\section{Chronotherapy based on home BP measurements in the morning}

The frequent occurrence of cardiovascular events in the morning is a well-known phenomenon that is associated with increases in ambulatory BP during the early morning, as well as changes in psychological and physiological indicators of stress [29, 30]. Although the presence of morning hypertension can be assessed by both ABPM and HBPM, the advantage of HBPM is that frequent assessment of morning BP control can be performed using home BP measurements which would not be feasible using ABPM devices. Assessment of morning hypertension is important, even in patients with well-controlled office BP. In the HONEST study, cardiovascular risk was increased in patients with morning home $\mathrm{BP}>145 \mathrm{~mm} \mathrm{Hg}$ and clinic BP $<130 \mathrm{~mm} \mathrm{Hg}$ (HR: $2.47,95 \%$ CI: 1.20 to 5.08 ) compared with patients with morning home $\mathrm{BP}<125 \mathrm{~mm} \mathrm{Hg}$ and clinic BP $<130 \mathrm{~mm} \mathrm{Hg}$ [20]. Therefore, efforts to control morning hypertension, even in patients with wellcontrolled office BP, are needed. Morning hypertension may be classified as the nondipper/riser (nocturnal hypertension) type, which is characterised by persistent high $\mathrm{BP}$ from night-time to morning, and is associated with increased risk to target organs and cardiovascular events. Morning hypertension may also be classified as morning surge, which is associated with extreme BP dipping at night-time and increased stroke risk. The Japan Morning
Surge-1 study investigated the effects of doxazosin add-on therapy at bedtime in treated hypertensive patients with selfmeasured morning SBP levels $>135 \mathrm{~mm} \mathrm{Hg}$ [31]. The addition of a bedtime dose of doxazosin significantly reduced both morning and evening BP as well as urinary albumin excretion rate compared with the control group who receive no add-on therapy. The success of chronotherapy is recognised in the American Diabetes Association guidelines, which specifically recommend the administration of $\geq 1$ antihypertensive medications at bedtime [32]. However, chronotherapy may affect drug adherence since some patients tend to forget the bedtime dose.

\section{Recommendation 6}

Titration should be based on targeting a mean home BP of $<135 / 85 \mathrm{~mm} \mathrm{Hg}$. However, in cases of high morning BP and normal evening BP, uptitration of drug treatment should be considered even if mean home $\mathrm{BP}$ is $<135 / 85 \mathrm{~mm} \mathrm{Hg}$. HBPM may aid chronotherapy of hypertension by helping identify those patients who experience isolated morning hypertension (Class IIa, Level of Evidence B).

\section{Application of home BP measurements into clinical hypertension guidelines}

The JSH 2014 guidelines for the management of hypertension offer a stepwise pathway to diagnosing hypertension that recognises a role for HBPM, both as an initial screening tool and following a clinic measure of $\geq 140 / 90$ $\mathrm{mm} \mathrm{Hg}$. A mean home BP measurement of $\geq 135 / 85 \mathrm{~mm} \mathrm{Hg}$ is recommended as providing a definitive diagnosis of hypertension [9]. The 2015 hypertension guidelines of the Taiwan Society of Cardiology (TSOC) and the Taiwan Hypertension Society (TSH) also include HBPM within their diagnostic algorithm [33]. The guidelines recommend treatment based on an office BP measurement of $\geq 140 / 90$ $\mathrm{mm} \mathrm{Hg}$ (or $\geq 130 / 80 \mathrm{~mm} \mathrm{Hg}$ in special patient groups) following confirmation of target organ damage. In patients with an office BP measurement of $\geq 140 / 90 \mathrm{~mm} \mathrm{Hg}$ without target organ damage, the TSOC/TSH guidelines recommend either HBPM or ABPM.

\section{Recommendation 7}

Given the utility of HBPM, the expert panel suggests that HBPM may be incorporated into local clinical hypertension guidelines. The pragmatic foreseen problems are availability and affordability. However, these can be overcome by health authorities through partial reimbursement or lending home BP devices to patients (Class IIa, Level of Evidence C). 


\section{Telemonitoring of home BP}

In addition to the use of nonvalidated devices, another potential disadvantage of HBPM is the inaccuracy of patient reports and the inability of physicians to derive any meaningful conclusions on the basis of handwritten patient BP logbooks. The combination of HBPM with teletransmission (telemonitoring) of patient data represents an opportunity to improve the accuracy and reliability of home BP reports. Moreover, the findings of a 6-month RCT showed that patient self-management of hypertension based on home BP teletransmission led to a better control of ambulatory BP than usual care involving office BP measurements [34]. The percentage of patients with daytime BP normalisation was higher with home-based teletransmission $(62 \%)$ compared with usual care $(50 \% ; P<0.05)$. Another RCT that compared usual care with self-measurement and transmission of $\mathrm{BP}$ readings to a secure website for review by a nurse or doctor showed a mean difference in daytime ambulatory BP of $4.3 / 2.3 \mathrm{~mm} \mathrm{Hg}$ that favoured telemonitoring [35]. However, the intervention was associated with increased use of healthcare resources. Finally, a meta-analysis investigating the clinical utility and cost effectiveness of home BP telemonitoring concluded that the use of telemonitoring is more costly compared with usual care, but does offer a useful tool for improved control of hypertension [36].

\section{Recommendation 8}

Information and communication technology (ICT)-based HBPM may be beneficial, especially for patients who live in remote Asian geographical locations. The expert panel agreed that that telemonitoring of home BP, which requires active participation by patients, may have an important role in clinical practice in the near future (Class IIb, Level of Evidence B).

\section{What home BP measurement devices should be used?}

The Chinese, Japanese, Korean, and European guidelines all recommend the use of validated HBPM devices $[9,10,14$, 15]. The Japanese and European guidelines specifically provide direction to websites that list available brachial BP measuring oscillometric devices that are validated for home use. However, these devices may not be practical for developing countries where the higher cost of validated devices may limit access. Nonvalidated devices are available, but there are questions regarding device accuracy. Another possibility for HBPM is the use of smartphonebased applications. A cross-sectional study evaluated the content of medical apps designed for hypertension management, with the top 107 apps analysed [37]. Although the majority of apps were designed for health management functions, $14 \%$ of Android apps could transform the smartphone into a medical device for measuring BP. However, none of these apps used a BP cuff or offered any validation against a gold standard.

\section{Recommendation 9}

Consistent with the need for medical device accuracy, the expert panel recommends that a validated brachial BP measuring oscillometric device should be used for measuring home BP (Class I, Level of Evidence C).

Where this is not feasible, for example due to the cost or limited access to the recommended device, the brachial BP measuring oscillometric device of choice should be calibrated every 6-12 months to ensure device accuracy (Class IIb, Level of Evidence C).

\section{Future perspectives}

As the value of HBPM is increasingly recognised in the literature and in national guidelines, its use will continue to rise in Asia, and HBPM should ultimately become part of the routine management of hypertension. The problems of cost and availability of home BP devices may be overcome by establishing borrowing systems for devices from village health workers or nearby clinics. Therefore, performing a series of home BP measurements for 3 to 7 days using the standard technique should be feasible for most patients. Average home BP should be determined first by averaging morning and night-time BP to see whether home BP is under control. This should then be followed by averaging morning home BP to investigate the presence of morning hypertension for further medication adjustment. These measures will allow the quality of $\mathrm{BP}$ control to be improved.

Successful utilisation of ICT-based HBPM has been demonstrated [35, 38], and is now available and set up in some medical centres to receive home BP transmission from registered patients. Immediate consultation can be made from those patients living in remote areas as well as poststroke or disabled patients without having to travel or being transferred to see their doctors. ICT-based HBPM may be particularly beneficial in Asia where many countries have large remote or rural populations [39], and further research is warranted.

Nocturnal hypertension detected via HBPM has been less well investigated, but studies have shown that it is associated with hypertensive organ damage [40, 41]. In the near future, basic home night-time BP monitoring devices should become more widely available. Nocturnal hypertension can be detected by this method [42], and thus may offer a more practical alternative to the $24 \mathrm{~h} \mathrm{ABPM}$. 


\section{Conclusions}

Although office BP measurement remains the cornerstone of hypertension management, HBPM is an important adjunct, providing accuracy in the diagnosis of hypertension through its ability to detect white-coat hypertension and masked hypertension, and thereby improving the appropriate management of disease. In addition to improving hypertension control, HBPM is superior to office BP measurements in predicting a future cardiovascular event. A key consideration in Asia is access to validated home BP devices, which often involve considerable out-of-pocket expense to the patient. Nonvalidated devices, while readily available, may only be used in exceptional circumstances. However, with constant technological improvements, it is anticipated that the cost of validated devices will continue to decrease. In concert with these advances, it is anticipated that patient self-care behaviours will improve and contribute to better diagnosis and treatment of hypertension. In the interim, reserving the use of home BP devices for those patients with high office BP in the absence of target organ damage and for patients with possible prehypertension based on office BP readings might be a suitable compromise.

The expert panel has developed a set of general recommendations for the use of HBPM. Where evidence originating from the region is currently absent, we are mindful that discussions arising from these recommendations will stimulate further research initiatives through which to inform subsequent iterations of the recommendations, with increased levels of evidence.

Funding The development of this article was made possible through a grant from the Korea Health Technology R\&D Project through the Korea Health Industry Development Institute (KHIDI), funded by the Ministry of Health \& Welfare, Republic of Korea (Grant Number: HI13C0715); and a grant from the Foundation for Development of the Community (Tochigi, Japan). Editorial and writing support was provided by Howard Christian and Chris Facey of MIMS (Hong Kong) Limited and was funded by Pfizer. The meeting during which these recommendations were formulated and discussed was made possible by an unrestricted educational grant from Pfizer. However, the recommendations were developed by the HOPE Asia Network independently of Pfizer.

\section{Compliance with ethical standards}

Conflict of interest $\mathrm{C}$-HC has received honoraria for serving as a speaker or member of a speaker bureau for AstraZeneca, Bayer AG, Boehringer Ingelheim, Bristol-Myers Squibb, Daiichi Sankyo, Merck \& Co, Novartis, Pfizer, Sanofi, Servier, and Takeda. Y-CC has received honoraria for serving as a speaker, advisor, or chair for Abbott, AstraZeneca, Bayer, Boehringer Ingelheim, GSK, Medtronic, MSD, Novartis, Pfizer, Reckitt Benckiser, Sanofi, Servier, and Takeda; and sponsorship to scientific conferences from Medtronic, Pfizer, Reckitt Benckiser, Servier, and Takeda. She is also the current President of the Malaysian Society of Hypertension, with an interest in promoting the use of home BP measurements. Omron Healthcare and other manufacturers of digital blood pressure devices have supported the society's annual scientific conference and several BP screening public health activities. KK has received research grants from A\&D Co., Bayer Yakuhin, Boehringer Ingelheim, Daiichi Sankyo, EA Pharma, Fukuda Denshi, Medtronic, Mitsubishi Tanabe Pharma Corporation, Mochida Pharmaceutical Co., Omron Healthcare, Otsuka, Pfizer, Takeda, and Teijin Pharma; and honoraria from Daiichi Sankyo, Omron Healthcare, and Takeda. SP has received honoraria from Astellas and Pfizer; and consultation fees from Takeda. SS has received honoraria from Bayer, Novartis, Pfizer, Sanofi Aventis, and Servier; and travel, accommodation, and conference registration support from Atco Pharmaceutical, Bayer, Highnoon Laboratories, Novartis, Pfizer, Sanofi Aventis, and Servier. GPS has received a research grant related to hypertension monitoring and treatment from Pfizer. YZ has received research grants from Bayer, Novartis, and Shuanghe; and lecture fees from Bayer, Daiichi Sankyo, MSD, Novartis, Pfizer, Sanofi, Servier, Shuanghe, and Takeda. The other authors declare that they have no conflict of interest.

Open Access This article is licensed under a Creative Commons Attribution 4.0 International License, which permits use, sharing, adaptation, distribution and reproduction in any medium or format, as long as you give appropriate credit to the original author(s) and the source, provide a link to the Creative Commons license, and indicate if changes were made. The images or other third party material in this article are included in the article's Creative Commons license, unless indicated otherwise in a credit line to the material. If material is not included in the article's Creative Commons license and your intended use is not permitted by statutory regulation or exceeds the permitted use, you will need to obtain permission directly from the copyright holder. To view a copy of this license, visit http://creativecommons. org/licenses/by/4.0/.

\section{References}

1. Kokubo Y. PL 02-2 Perspective on elderly hypertension in Asia. J Hypertens. 2016;34:e182.

2. Mills KT, Bundy JD, Kelly TN, Reed JE, Kearney PM, Reynolds $\mathrm{K}$, et al. Global disparities of hypertension prevalence and control: a systematic analysis of population-based studies from 90 countries. Circulation. 2016;134:441-50.

3. Castillo R. SSA 03-1 Prevalence and management of hypertension in Southeast Asia. J Hypertens. 2016;34:e4.

4. Suh IPL. 01-2 Blood pressure and cardiovascular disease mortality in the Asia Pacific region. J Hypertens. 2016;34:e11.

5. Kario K. Proposal of a new strategy for ambulatory blood pressure profile-based management of resistant hypertension in the era of renal denervation. Hypertens Res. 2013;36:478-84.

6. Angeli F, Reboldi G, Verdecchia P. The 2014 hypertension guidelines: implications for patients and practitioners in Asia. Heart Asia. 2015;7:21-25.

7. Sheppard JP, Schwartz CL, Tucker KL, McManus RJ. Modern management and diagnosis of hypertension in the United Kingdom: home care and self-care. Ann Glob Health. 2016;82:274-87.

8. Mancia G, Fagard R, Narkiewicz K, Redón J, Zanchetti A, Böhm $\mathrm{M}$, et al. $2013 \mathrm{ESH} / \mathrm{ESC}$ Guidelines for the management of arterial hypertension: the Task Force for the management of arterial hypertension of the European Society of Hypertension (ESH) and of the European Society of Cardiology (ESC). J Hypertens. 2013;31:1281-357.

9. Shimamoto K, Ando K, Fujita T, Hasebe N, Higaki J, Horiuchi M, et al. The Japanese Society of Hypertension guidelines for the 
management of hypertension (JSH 2014). Hypertens Res. 2014;37:253-390.

10. Shin J, Park JB, Kim K, Kim JH, Yang DH, Pyun WB, et al. 2013 Korean Society of Hypertension guidelines for the management of hypertension: part I - epidemiology and diagnosis of hypertension. Clin Hypertens. 2015;21:1.

11. Chia YC, Buranakitjaroen $\mathrm{P}$, Chen $\mathrm{CH}$, Divinagracia R, Hoshide $\mathrm{S}$, Park S, et al. Current status of home blood pressure monitoring in Asia - statement from the HOPE Asia Network.Clin Hypertens. 2017;19:1192-201.

12. Banegas JR, Segura J, Sobrino J, Rodriguez-Artalejo F, de la Sierra A, de la Cruz JJ, et al. Effectiveness of blood pressure control outside the medical setting. Hypertension. 2007;49:62-68.

13. Hodgkinson J, Mant J, Martin U, Guo B, Hobbs FD, Deeks JJ, et al. Relative effectiveness of clinic and home blood pressure monitoring compared with ambulatory blood pressure monitoring in diagnosis of hypertension: systematic review. BMJ. 2011;342: d3621.

14. Parati G, Stergiou GS, Asmar R, Bilo G, de Leeuw P, Imai Y, et al. European Society of Hypertension practice guidelines for home blood pressure monitoring. J Hum Hypertens. 2010;24:779-85.

15. Wang JG, for the China HBPM Consensus Expert Panel. Home blood pressure monitoring: a China consensus document. Chin J Hypertens. 2012;20:525-9.

16. Niiranen TJ, Johansson JK, Reunanen A, Jula AM. Optimal schedule for home blood pressure measurement based on prognostic data: the Finn-Home study. Hypertension. 2011;57:1081-6.

17. Ohkubo T, Asayama K, Kikuya M, Metoki H, Hoshi H, Hashimoto J, et al. How many times should blood pressure be measured at home for better prediction of stroke risk? Ten-year follow-up results from the Ohasama study. J Hypertens. 2004;22:1099-104.

18. Tientcheu D, Ayers C, Das SR, McGuire DK, de Lemos JA, Khera A, et al. Target organ complications and cardiovascular events associated with masked hypertension and white coat hypertension: analysis from the Dallas Heart Study. J Am Coll Cardiol. 2015;66:2159-69.

19. Ohkubo T, Imai Y, Tsuji I, Nagai K, Kato J, Kikuchi N, et al. Home blood pressure measurement has a stronger predictive power for mortality than does screening blood pressure measurement: a population-based observation in Ohasama, Japan. J Hypertens. 1998;16:971-5.

20. Kario K, Saito I, Kushiro T, Teramukai S, Ishikawa Y, Mori Y, et al. Home blood pressure and cardiovascular outcomes in patients during antihypertensive therapy: primary results of HONEST, a large-scale prospective, real-world observational study. Hypertension. 2014;64:989-96.

21. Kario K, Saito I, Kushiro T, Teramukai S, Tomono Y, Okuda Y, et al. Morning home blood pressure is a strong predictor of coronary artery disease: the HONEST Study. J Am Coll Cardiol. 2016;67:1519-27.

22. Hoshide S, Yano Y, Haimoto $H$, Yamagiwa K, Uchiba K, Nagasaka S, et al. Morning and evening home blood pressure and risks of incident stroke and coronary artery disease in the Japanese general practice population: the Japan Morning Surge-Home Blood Pressure study. Hypertension. 2016;68:54-61.

23. Niiranen TJ, Hanninen M-R, Johansson J, Reunanen A, Jula AM. Home-measured blood pressure is a stronger predictor of cardiovascular risk than office blood pressure: the Finn-Home study. Hypertension. 2010;55:1346-51.

24. European Society of Hypertension/European Society of Cardiology. 2013 practice guidelines for the management of arterial hypertension of the European Society of Hypertension (ESH) and the European Society of Cardiology (ESC): ESH/ESC Task Force for the Management of Arterial Hypertension. J Hypertens. 2013;31:1925-38.
25. Staessen JA, Hond ED, Celis H, Fagard R, Keary L, Vandenhoven $\mathrm{G}$, et al. Antihypertensive treatment based on blood pressure measurement at home or in the physician's office: a randomized controlled trial. JAMA. 2004;291:955-64.

26. Marquez-Contreras E, Martell-Claros N, Gil-Guillen V, de la Figuera-Von Wichmann M, Casado-Martinez JJ, Martin-de Pablos JL, et al. Efficacy of a home blood pressure monitoring programme on therapeutic compliance in hypertension: the EAPACUM - HTA study. J Hypertens. 2006;24:169-75.

27. Agarwal R, Bills JE, Hecht TJW, Light RP. Role of home blood pressure monitoring in overcoming therapeutic inertia and improving hypertension control: a systematic review and metaanalysis. Hypertension. 2011;57:29-38.

28. McManus RJ, Mant J, Haque MS, Bray EP, Bryan S, Greenfield $\mathrm{SM}$, et al. Effect of self-monitoring and medication self-titration on systolic blood pressure in hypertensive patients at high risk of cardiovascular disease: the TASMIN-SR randomized clinical trial. JAMA. 2014;312:799-808.

29. Kario K. Time for focus on morning hypertension: pitfall of current antihypertensive medication. Am $\mathrm{J}$ Hypertens. 2005;18:149-51.

30. Wang JG, Kario K, Park JB, Chen CH Morning blood pressure monitoring in the management of hypertension. J Hypertens.2017;35:1554-63.

31. Kario K, Matsui Y, Shibasaki S, Eguchi K, Ishikawa J, Hoshide S, et al. An alpha-adrenergic blocker titrated by self-measured blood pressure recordings lowered blood pressure and microalbuminuria in patients with morning hypertension: the Japan Morning Surge-1 Study. J Hypertens. 2008;26:1257-65.

32. American Diabetes Association. Standards of medical care in diabetes 2017. Diabetes Care. 2017;40:S1-S135.

33. Chiang C-E, Wang T-D, Ueng K-C, Lin T-H, Yeh H-I, Chen C-Y, et al. 2015 guidelines of the Taiwan Society of Cardiology and the Taiwan Hypertension Society for the management of hypertension. J Chin Med Assoc. 2015;78:1-47.

34. Parati G, Omboni S, Albini F, Piantoni L, Giuliano A, Revera M, et al. Home blood pressure telemonitoring improves hypertension control in general practice: the TeleBPCare study. J Hypertens. 2009;27:198-203.

35. McKinstry B, Hanley J, Wild S, Pagliari C, Paterson M, Lewis S, et al. Telemonitoring based service redesign for the management of uncontrolled hypertension: multicentre randomised controlled trial. BMJ. 2013;346:f3030.

36. Omboni S, Gazzola T, Carabelli G, Parati G. Clinical usefulness and cost effectiveness of home blood pressure telemonitoring: meta-analysis of randomized controlled studies. J Hypertens. 2013;31:455-67.

37. Kumar N, Khunger M, Gupta A, Garg N. A content analysis of smartphone-based applications for hypertension management. J Am Soc Hypertens. 2015;92:130-6.

38. Kario K, Tomitani N, Kanegae H, Ishii H, Uchiyama K, Yamagiwa $\mathrm{K}$, et al. Comparative effects of an angiotensin II receptor blocker (ARB)/diuretic vs. ARB/calcium-channel blocker combination on uncontrolled nocturnal hypertension evaluated by Information and Communication Technology-Based Nocturnal Home Blood Pressure Monitoring - The NOCTURNE Study.Circ J.2017;81:948-57.

39. Kaihara T, Eguchi K, Kario K. Home BP monitoring using a telemonitoring system is effective for controlling BP in a remote island in Japan. J Clin Hypertens (Greenwich). 2014;16: 814-9.

40. Ishikawa J, Hoshide S, Eguchi K, Ishikawa S, Shimada K, Kario $\mathrm{K}$, et al. Nighttime home blood pressure and the risk of hypertensive target organ damage. Hypertension. 2012;60:921-8.

41. Kario K, Hoshide S, Haimoto H, Yamagiwa K, Uchiba K, Nagasaka S, et al. Sleep Blood Pressure Self-Measured at Home 
as a Novel Determinant of Organ Damage: Japan Morning Surge Home Blood Pressure (J-HOP) Study. J Clin Hypertens (Greenwich). 2015;17:340-8.
42. Kario K. Essential Manual of 24-hour Blood Pressure Management from Morning to Nocturnal Hypertension. London: WileyBlackwell; 2015. 